\title{
ON A FAMILY OF ANALYTIC DISCS ATTACHED TO A REAL SUBMANIFOLD $M \subset \mathbb{C}^{N+1 *}$
}

\author{
VALENTIN BURCEA ${ }^{\dagger}$
}

\begin{abstract}
We construct a family of analytic discs attached to a real submanifold $M \subset \mathbb{C}^{N+1}$ of codimension 2 near a $\mathrm{CR}$ singularity. These discs are mutually disjoint and form a smooth hypersurface $\widetilde{M}$ with boundary $M$ in a neighborhood of the CR singularity. As an application we prove that if $p$ is a flat-elliptic CR singularity and if $M$ is nowhere minimal at its CR points and does not contain a complex manifold of dimension $(n-2)$, then $\widetilde{M}$ is a smooth Levi-flat hypersurface. Moreover, if $M$ is real analytic we obtain that $\widetilde{M}$ is real-analytic across the boundary manifold $M$.
\end{abstract}

Key words. CR singularity, hull of holomorphy, analytic disc.

AMS subject classifications. $32 \mathrm{~V} 40$.

1. Introduction and main results. Let $M \subset \mathbb{C}^{N+1}$ be a real submanifold. A point $p \in M$ is called a CR singularity if it is a jump discontinuity point of the map $M \ni q \mapsto \operatorname{dim}_{\mathbb{C}} T_{q}^{0,1} M$ defined near $p$. Here $T_{q}^{0,1} M$ is the CR tangent space to $M$ at $q$.

We assume that $\operatorname{codim}_{\mathbb{R}} M=2$. Bishop considered the case when there exist $(z, w)$ holomorphical coordinates in $\mathbb{C}^{2}$, such that near the $\mathrm{CR}$ singularity $p=0$ the submanifold $M \subset \mathbb{C}^{2}$ is defined by

$$
w=z \bar{z}+\lambda\left(z^{2}+\bar{z}^{2}\right)+\mathrm{O}(3)
$$

where $\lambda \in[0, \infty)$ is a holomorphic invariant called the Bishop invariant. In the case when $\lambda \in\left[0, \frac{1}{2}\right)$, Kenig-Webster proved in [14] the existence of an unique family of 1-dimensional analytic disks shrinking to the CR singularity $p=0$. The real-analytic case was studied by Huang-Krantz in [7].

Let $\left(z_{1}, \ldots, z_{N}, w\right)$ be the coordinates from $\mathbb{C}^{N+1}$. In this paper, we consider the higher dimensional case of (1.1) when the submanifold $M \subset \mathbb{C}^{N+1}$ is defined near $p=0$ by

$$
w=z_{1} \bar{z}_{1}+\lambda\left(z_{1}^{2}+\bar{z}_{1}^{2}\right)+Q\left(z_{1}, \bar{z}_{1}, z_{2}, \bar{z}_{2}, \ldots, z_{N}, \bar{z}_{N}\right)+\mathrm{O}(3),
$$

where $Q\left(z_{1}, \bar{z}_{1}, z_{2}, \bar{z}_{2}, \ldots, z_{N}, \bar{z}_{N}\right)$ is a quadratic form depending on $z_{2}, \bar{z}_{2}, \ldots, z_{N}, \bar{z}_{N}$ and combinations between $z_{2}, \bar{z}_{2}, \ldots, z_{N}, \bar{z}_{N}$ and $z_{1}, \bar{z}_{1}$. We say that $\lambda$ is elliptic if $\lambda \in\left[0, \frac{1}{2}\right)$.

In this paper we extend Kenig-Webster's Theorem from [14]. We prove the following result:

THEOREM 1.1. Let $M \subset \mathbb{C}^{N+1}$ be a smooth submanifold defined locally near $p=0$ by (1.2) such that $\lambda$ is elliptic. Then there exists a family of regularly embedded analytic discs with boundaries on $M$ that are mutually disjoint and that forms a smooth hypersurface $\widetilde{M}$ with boundary $M$ in a neighborhood of the $C R$ singularity $p=0$.

Then $\widetilde{M}$ given by Theorem 1.1 is not necessary a Levi-flat hypersurface as in Kenig-Webster's case from $[14]$ in $\mathbb{C}^{2}$.

*Received May 30, 2012; accepted for publication April 2, 2013. This project was supported by Science Foundation Ireland grant 10/RFP/MTH2878.

†School of Mathematics, Trinity College Dublin, Dublin 2, Ireland (valentin@maths.tcd.ie). 
The existence problem of a Levi-flat hypersurface with prescribed boundary $S$ in $\mathbb{C}^{N+1}$ with $N \geq 2$, was studied by Dolbeault-Tomassini-Zaitsev in [2] under the following natural assumptions on $S$ :

(i) $S$ is compact, connected and nowhere minimal at its CR points;

(ii) $S$ does not contain a complex submanifold of dimension $(n-2)$;

(iii) $S$ contains a finite number of flat elliptic CR singularities.

We would like to mention that properties of nowhere minimal CR submanifolds were studied by Lebl in [20].

The CR singularity $p=0$ is called elliptic if the quadratic part from (1.2) is positive definite. We say that $p=0$ is a "flat" if Definition 2.1 from [2] is satisfied. Under the precedent natural assumptions, Dolbeault-Tomassini-Zaitsev proved the existence of a (possibly singular) Levi-flat hypersurface which bounds $S$ in the sense of currents (see Theorem 1.3, [2]).

The graph case was studied by Dolbeault-Tomassini-Zaitsev in [3]: Let $\mathbb{C}^{N+1}=$ $\left(\mathbb{C}_{z}^{N} \times \mathbb{R}_{u}\right) \times \mathbb{R}_{v}$, where $w=u+i v$, and let $\Omega$ be a bounded strongly convex domain of $\mathbb{C}_{z}^{N} \times \mathbb{R}_{u}$ with smooth boundary $b \Omega$. Let $S \subset \mathbb{C}^{N+1}, n \geq 3$, be the graph of a function $g: b \Omega \longrightarrow \mathbb{R}_{v}$ such that $S$ satisfies the natural assumptions (i), (ii), (iii). Under these assumptions Dolbeault-Tomassini-Zaitsev proved the following result

THEOREM 1.2. Let $q_{1}, q_{2} \in b \Omega$ be the projections of the complex points $p_{1}, p_{2}$ of $S$, respectively. Then, there exists a Lipschitz function $f: \bar{\Omega} \longrightarrow \mathbb{R}_{v}$ which is smooth on $\bar{\Omega}-\left\{q_{1}, q_{2}\right\}$ and such that $\left.f\right|_{b \Omega}=g$ and $N=\operatorname{graph}(f)-S$ is a Levi-flat hypersurface of $\mathbb{C}^{N+1}$. Moreover, each complex leaf of $M_{0}$ is the graph of a holomorphic function $\varphi: \Omega^{\prime} \longrightarrow \mathbb{C}$ where $\Omega^{\prime} \subset \mathbb{C}^{n-1}$ is a domain with smooth boundary (that depends on the leaf) and $\varphi$ is smooth on $\Omega^{\prime}$.

As an application of Theorem 1.1 , we solve an open problem regarding the regularity of $f$ given by Theorem 1.2 at $q_{1}, q_{2}$, proposed by Dolbeault-TomassiniZaitsev in [3].

By combining Theorem 1.1 and Theorem 1.2 we obtain the following result

TheOREM 1.3. Let $M \subset \mathbb{C}^{N+1}$ be a smooth submanifold as in Theorem 1.2. Suppose $p$ is a point in $M$ such that $M$ is defined near $p=0$ by (1.2) satisfying the condition that (i) $p=0$ is a flat-elliptic CR singularity (ii) any CR point of $M$ near $p=0$ is non-minimal, and (iii) $M$ does not contain a complex submanifold of dimension $n-2$. Then $\widetilde{M}$ constructed by Theorem 1.1 is a smooth Levi-flat hypersurface with boundary $M$ in a neighborhood of $p=0$.

In the real analytic case our smoothness result combined with an similar argument as in the paper [13] of Huang-Yin concerning the analyticity of the local hull of holomorphy, gives the following result:

THEOREM 1.4. Let $M \subset \mathbb{C}^{N+1}$ be a real analytic submanifold defined near $p=0$ by (1.2) and that satisfies the assumptions of Theorem 1.3. Then $\widetilde{M}$ is a Levi-flat hypersurface real-analytic across the boundary manifold $M$.

We prove our results by following the lines of developed by Huang in [8], KenigWebster in [14], [15] and in particulary the construction of analytic discs developed by Huang-Krantz in [7]. First, we make a perturbation along the CR singularity and then we find a holomorphic change of coordinates depending smoothly on a parameter. Then, we will adapt the methods used in $\mathbb{C}^{2}$ by Huang-Krantz and Kenig-Webster in our case. 
We would like to mention that versions of our result were obtained in a higher codimensional case by Huang in [8] and Kenig-Webster in [15].

The study of CR singular real submanifolds in the complex space requires different methods than the case of $\mathrm{CR}$ manifolds. We mention here the problem of finding a normal form for a real submanifold in $\mathbb{C}^{2}$ defined by (1.1). For $\lambda$ non-exceptional, this problem was solved by Moser-Webster in [18]. When $\lambda>\frac{1}{2}$, Gong studied in [4] the existence of the real-analytic surfaces that are formally equivalent, but not biholomorphically equivalent to quadrics. The case $\lambda=0$ was initiated by Moser in [17] and well understood by Huang-Yin in the nice paper [11]. We would like to mention here also the paper [10] of Huang-Yin where Moser's Theorem from [17] is generalized to the higher dimensional case.

Acknowledgements. I am grateful to my supervisor Prof. Dmitri Zaitsev for useful conversations and for bringing me this problem to my attention. I would like thank the referee for helpful comments on the previous version of the manuscript.

\section{Preliminaries.}

2.1. A perturbation along the $\mathbf{C R}$ singularity. Let $\Delta$ be the unit open disc from $\mathbb{C}$ and let $S^{1}$ be its boundary. A map $f: \bar{\Delta} \longrightarrow \mathbb{C}^{N+1}$ is called an analytic disc if $\left.f\right|_{\Delta}$ is continuous and $\left.f\right|_{\Delta}$ is nonconstant and holomorphic. We say that $f$ is an analytic disc attached to $M$ if $f\left(\mathrm{~S}^{1}\right) \subset M$. We construct analytic discs attached to $M$ depending smoothly on

$$
X=\left(z_{2}, \ldots, z_{N}\right)=\left(x_{2}+i y_{2}, \ldots, x_{N}+i y_{N}\right) \approx 0 \in \mathbb{C}^{N-2} .
$$

By using the notation $z=z_{1}$, our manifold $M$ is defined near $p=0$ by

$$
w=z \bar{z}+\lambda\left(z^{2}+\bar{z}^{2}\right)+Q\left(z_{1}, \bar{z}_{1}, z_{2}, \bar{z}_{2}, \ldots, z_{N}, \bar{z}_{N}\right)+\mathrm{O}(3),
$$

or equivalently by

$$
\begin{aligned}
w= & H_{0,0}(X)+\bar{z} H_{0,1}(X)+z H_{1,0}(X)+z \bar{z}\left(1+H_{1,1}(X)\right) \\
& +\left(\lambda+H_{2,0}(X)\right) z^{2}+\left(\lambda+H_{0,2}(X)\right) \bar{z}^{2}+\mathrm{O}\left(|z|^{3}\right),
\end{aligned}
$$

where $H_{0,0}(X), H_{1,0}(X), H_{0,1}(X), H_{1,1}(X), H_{2,0}(X), H_{0,2}(X)$ are smooth functions vanishing at $X=0$.

We prove the following lemma:

Lemma 2.1. Let $M \subset \mathbb{C}^{2}$ be a real smooth submanifold defined near $p=0$ by $w=a z+b \bar{z}+O\left(|z|^{2}\right)$. Then

$$
T_{0}^{c} M \neq \emptyset \Longleftrightarrow b=0 .
$$

Proof. We need to solve the equations $\partial f=\bar{\partial} f=0$ at the point $z=w=0$. We compute:

$$
\left.\partial f\right|_{0}=\frac{\partial f}{\partial z}(0) d z+\frac{\partial f}{\partial w}(0) d w=-d w+a d z,\left.\quad \bar{\partial} f\right|_{0}=\frac{\partial f}{\partial \bar{z}}(0) d \bar{z}+\frac{\partial f}{\partial \bar{w}}(0) d \bar{w}=b d \bar{z} .
$$

We obtain $a d z=d w$ and $b d \bar{z}=0$. It follows that $p=0$ is a CR singularity if and only if $b=0$. 
We make a change of coordinates depending smoothly on $X \approx 0 \in \mathbb{C}^{N-2}$ preserving the CR singularity $p=0$ :

Proposition 2.2. There exists a biholomorphic change of coordinates in $(z, w)$ depending smoothly on $X \approx 0 \in \mathbb{C}^{N-2}$ that sends (2.3) to a submanifold defined by

$$
w=z \bar{z}+\lambda(X)\left(z^{2}+\bar{z}^{2}\right)+O\left(|z|^{3}\right),
$$

preserving the $C R$ singularity $p=0$. Here $0 \leq \lambda(X)<\frac{1}{2}$ for $X \approx 0 \in \mathbb{C}^{N-2}$ and $\lambda(0)=\lambda$.

Proof. We consider a local defining function for $M$ near $p=0$

$$
\begin{aligned}
f(z, X, w)= & -w+H_{0,0}(X)+\bar{z} H_{0,1}(X)+z H_{1,0}(X)+z \bar{z}\left(1+H_{1,1}(X)\right) \\
& +\left(\lambda+H_{2,0}(X)\right) z^{2}+\left(\lambda+H_{0,2}(X)\right) \bar{z}^{2}+\mathrm{O}\left(|z|^{3}\right) .
\end{aligned}
$$

Each fixed $X \approx 0 \in \mathbb{C}^{N-2}$ defines us a real submanifold in $\mathbb{C}^{2}$ which may not have a CR singularity at the point $z=w=0$ because $H_{0,1}(X)$ may be different than 0 (see Lemma 2.1). Therefore we need to make a change of coordinates in $(z, w)$ depending smoothly on $X \approx 0 \in \mathbb{C}^{N-2}$ that perturbs the CR singularity $p=0$. We consider the following equation

$$
0=\frac{\partial f}{\partial \bar{z}}=H_{0,1}(X)+\left(1+H_{1,1}(X)\right) z+B(z, \bar{z}, X),
$$

where $B(z, \bar{z}, X)$ is a smooth function. Since $H_{1,1}(0)=0$, by applying the implicit function theorem we obtain a smooth solution $z_{0}=z_{0}(X)$ for $(2.8)$. By making the translation $\left(w^{\prime}, z^{\prime}\right)=\left(w, z+z_{0}(X)\right)$, the equation (2.3) becomes

$$
\begin{aligned}
w= & z C_{1,0}(X)+z \bar{z}\left(1+C_{1,1}(X)\right)+\left(\lambda+C_{2,0}(X)\right) z^{2} \\
& +\left(\lambda+C_{0,2}(X)\right) \bar{z}^{2}+\mathrm{O}\left(|z|^{3}\right)
\end{aligned}
$$

where $C_{1,0}(X), C_{1,1}(X), C_{2,0}(X), C_{0,2}(X)$ are smooth functions vanishing at $X=0$. Let $\gamma(X)=1+C_{1,1}(X), \Lambda_{1}(X)=\lambda+C_{2,0}(X), \Lambda_{2}(X)=\lambda+C_{0,2}(X)$. In the new coordinates $(w, z):=\left(\left(w-C_{1,0}(X) z\right) / \gamma(X), z\right)$, the equation (2.9) becomes

$$
w=z \bar{z}+\Lambda_{1}(X) z^{2}+\Lambda_{2}(X) \bar{z}^{2}+\mathrm{O}\left(|z|^{3}\right)
$$

Next, we consider a map $\Theta(X)$ such that $\Lambda_{2}(X) e^{-2 i \Theta(X)} \geq 0$. Changing the coordinates $(w, z):=\left(w, z e^{i \Theta(X)}\right)$, we can assume $\Lambda_{2}(X) \geq 0$. Changing again the coordinates $(w, z):=\left(w+\left(\Lambda_{1}(X)-\Lambda_{2}(X)\right) z^{2}, z\right)$ we obtain (2.6).

We write

$$
M: w=z \bar{z}+\lambda(X)\left(z^{2}+\bar{z}^{2}\right)+P(z, X)+i K(z, X),
$$

where $P(z, X)$ and $K(z, X)$ are real smooth functions. We prove an extension of Lemma 1.1 from [14]:

Proposition 2.3. There exists a holomorphic change of coordinates in $(z, w)$ depending smoothly on $X \approx 0 \in \mathbb{C}^{N-2}$ in which $K$ and its partial derivatives in $z$ and $\bar{z}$ of order less or equal to $l$ vanish at $z=0$. 
Proof. By making the substitution $\left(z^{\prime}(X), w^{\prime}(X)\right)=(z, w+B(z, X, w))$ and by (2.11) it follows that

$$
M: w^{\prime}=q(z, X)+P(z, X)+i K(z, X)+\operatorname{Re} B(z, X, w)+i \operatorname{lm} B(z, X, w),
$$

where $q(z, X)=z \bar{z}+\lambda(X)\left(z^{2}+\bar{z}^{2}\right)$. We want to make the derivatives in $z$ of order less than $l$ of $i(K(z, X)+\operatorname{Im} B(z, X, w))$ vanish at $z=0$. By multiplying (2.12) by $i=\sqrt{-1}$, our problem is reduced to the following general equation

$$
\operatorname{Re} B(z, X, q(z, X)+P(z, X)+i K(z, X))=f(z, \bar{z}, X),
$$

where $f(z, \bar{z}, X)$ is a real formal power series in $(z, \bar{z}, X)$ with cubic terms in $z$ and $\bar{z}$ with coefficients depending smoothly on $X \approx 0 \in \mathbb{C}^{N-2}$. We write

$$
\begin{aligned}
f(z, \bar{z}, X) & =\sum_{m=3}^{l} f_{m}(z, \bar{z}, X), f_{m}(z, \bar{z}, X) \\
& =\sum_{j_{1}+j_{2}=m} c_{j_{1}, j_{2}}^{m}(X) z^{j_{1}} \bar{z}^{j_{2}}, c_{j_{1}, j_{2}}^{m}(X)=\overline{c_{j_{2}, j_{1}}^{m}(X)}, \\
B(z, X, w) & =\sum_{m=3}^{l} B_{m}(z, X, w), \quad B_{m}(z, X, w)=\sum_{j_{1}+2 j_{2}=m} b_{j_{1}, j_{2}}^{m}(X) z^{j_{1}} w^{j_{2}} .
\end{aligned}
$$

We solve inductively (2.13) by using the following lemma:

Lemma 2.4. The equation (2.13) has a unique solution with the normalization condition $\operatorname{Im} B_{m}(0, X, u)=0$.

Proof. We define the weight of $z$ to be 1 and the weight of $w$ to be 2 . We say that the polynomial $B_{m}(z, X, w)$ has weight $m$ if $B_{m}\left(t z, X, t^{2} w\right)=t^{m} B_{m}(z, X, w)$. Let $\mathbb{B}_{m}$ be the space of all such homogeneous holomorphic polynomials in $(z, w)$ of weight $m$ satisfying the normalization condition with coefficients depending smoothly on $X \approx 0 \in \mathbb{C}^{N-2}$ and let $\mathbb{F}_{m}$ be the space of all homogeneous polynomials $f_{m}(z, \bar{z}, X)$ of bidegree $(k, l)$ in $(z, \bar{z})$ with $k+2 l=m$ with coefficients depending smoothly on $X \approx 0 \in \mathbb{C}^{N-2}$. We can rewrite (2.13) as follows

$$
B_{m}(z, X, q(z, X)+P(z, X)+i K(z, X))=B_{m}(z, X, q(z, X))+\mathrm{O}\left(|z|^{m+1}\right) .
$$

In order to solve (2.15) it is enough to prove that we have a linear invertible transformation

$$
\varphi(X): \mathbb{B}_{m} \ni B_{m}(z, X, w) \mapsto \operatorname{Re} B_{m}(z, X, q(z, X)) \in \mathbb{F}_{m},
$$

depending smoothly on $X \approx 0 \in \mathbb{C}^{N-2}$. By Lemma 1.1 of the paper [14] of KenigWebster, it follows that $\varphi(X)$ is invertible for $X=0 \in \mathbb{C}^{N-2}$. By the continuity it follows that $\varphi(X)$ is invertible. If it is necessary we shrink the range of $X \approx 0 \in$ $\mathbb{C}^{N-2}$.

The proof is completed now by induction and by using Lemma 2.4. 
2.2. Preliminary preparations. Let $w=u+i v$ and $I_{\epsilon}:=(-\epsilon, \epsilon) \subset \mathbb{R}$, for $0<\epsilon<<1$. We assume that $M$ is defined by (2.11) and satisfies the properties of Proposition 2.3.

In order to define a family of attached discs to the manifold $M$, we define the following domain

$$
D_{X, r}=\{z \in \mathbb{C} ; v=0, q(z, X)+P(z, X) \leq u<\epsilon\},
$$

where $u=r^{2}$. By similar arguments as in the paper [8] of Huang, it follows that $D_{X, r}$ is a simply connected bounded set of $\mathbb{C}$. Therefore there exists a unique mapping $r \sigma_{X, r}: \Delta \rightarrow D_{X, r}$ such that $\sigma_{X, r}(0)=0$ and $\sigma_{X, r}^{\prime}(0)>0$. Then, for $0<r<<1$ we can define the following family of curves depending smoothly on $X \approx 0 \in \mathbb{C}^{N-2}$

$$
\gamma_{X, r}=\left\{z \in \mathbb{C} ; q(z, X)+P(z, X)=r^{2}\right\} \text {. }
$$

Next, we define the following family of analytic discs

$$
\left\{\left(r \sigma_{X, r}, X, r^{2}\right)\right\}_{X \approx 0 \in \mathbb{C}^{N-2}, 0<r<<1} .
$$

The family of analytic discs shrinks to $\{0\} \times \mathcal{O} \times\{0\}$ as $r \mapsto 0$, where $0 \in \mathcal{O} \subset \mathbb{C}^{N-2}$ and fills up the following domain

$$
\widetilde{M}_{0}=\left\{(z, X, u) \in \mathbb{C} \times \mathbb{C}^{N-2} \times \mathbb{R} ;\|X\|<<1, q(z, X)+P(z, X) \leq u\right\} .
$$

2.3. The Hilbert transform on a variable curve. Let $\gamma_{X, r}$ given by (2.18), where $r$ is taken very small. For a function $\varphi_{X, r}(\theta)$ defined on $\gamma_{X, r}$ we define its Hilbert transform $\mathrm{H}_{X, r}\left[\varphi_{X, r}\right]$ to be the boundary value of a function holomorphic inside $\gamma_{X, r}$, with its imaginary part vanishing at the origin. For more informations about the Hilbert transform we mention here the book [5] of Helmes.

For $\alpha \in(0,1)$ we define the following Banach spaces:

$$
\begin{gathered}
\mathcal{C}^{\alpha}:=\left\{u: \gamma_{X, r} \longrightarrow \mathbb{R} ;\|u\|_{\alpha}:=\sup _{\substack { x \in \gamma_{X}, r \\
\begin{subarray}{c}{x, y \in \gamma_{X}, r \\
x \neq y{ x \in \gamma _ { X } , r \\
\begin{subarray} { c } { x , y \in \gamma _ { X } , r \\
x \neq y } }\end{subarray}} \frac{|u(x)-u(y)|}{|x-y|^{\alpha}}<\infty\right\}, \\
\mathcal{C}^{k, \alpha}:=\left\{u: \gamma_{X, r} \longrightarrow \mathbb{R} ;\|u\|_{k, \alpha}:=\sum_{|\beta| \leq k}\left\|\mathrm{D}^{\beta} u\right\|_{\alpha}<\infty\right\} .
\end{gathered}
$$

Let $X:=\left\{x_{2}, y_{2}, \ldots, x_{N}, y_{N}\right\}$. The following result can be proved by using the same lines as in Kenig-Webster's paper [14] (Theorem 2.5) or from Kenig-Webster's paper [15]:

Proposition 2.5. As $r \rightarrow 0$ and $X \approx 0 \in \mathbb{C}^{N-2}$ we have

$$
\begin{aligned}
\left\|\mathcal{H}_{X, r}\right\|_{j, \alpha} & =O(r), \text { for all } j \leq l-2 ; \quad\left\|\left(\partial_{X}^{|I|} \partial_{r}^{s}\right) \mathcal{H}_{X, r}\right\|_{j, \alpha} \\
& =O(1), \text { for all } j+2 s \leq l-4, I \in \mathbb{N}^{N-2} .
\end{aligned}
$$


2.4. An implicit functional equation. During this section we work in the Holder space $\left(\mathcal{C}^{j, \alpha},\|\cdot\|_{j, \alpha}\right)$. We employ ideas developed by Huang-Krantz in [7], Huang in [8], Kenig-Webster in [14], [15] and we define the following auxiliary hypersurface

$$
M_{0}=\left\{(z, X, u) \in \mathbb{C} \times \mathbb{C}^{N-2} \times \mathbb{R} ;\|X\|<<1, q(z, X)+P(z, X)=u<\epsilon\right\},
$$

where $\epsilon>0$ is small enough and $w=u+i v$. We would like to find a map of the following type

$$
T=T[X]:=(z(1+\mathcal{F}(z, X, r)), \mathcal{B}(z, X, r))
$$

such that $T\left(M_{0}\right) \subseteq M$. Here $\mathcal{F}, \mathcal{B}$ are holomorphic functions in $z$ and smooth in $(X, r)$. It follows that

$$
\left.\mathcal{B}(z, X, r)\right|_{\gamma_{X, r}}=\left.(q+P+i K)(z+z \mathcal{F}(z, X, r), X)\right|_{\gamma_{X, r}},
$$

where $\gamma_{X, r}$ is the curve defined by (2.18). By using the Hilbert transform on the curve $\gamma_{X, r}$ and by dividing by $r^{2}$ the equation (2.25), it follows that there exists a smooth function $V(X, r)$ such that

$$
\begin{gathered}
\left.q(z(1+\mathcal{F}(z, X, r)), X)\right|_{\gamma_{X, r}}=-\left.P(z(1+\mathcal{F}(z, X, r)), X)\right|_{\gamma_{X, r}} \\
-\left.\mathcal{H}_{X, r}[K(z(1+\mathcal{F}(z, X, r)), X)]\right|_{\gamma_{X, r}}+V(X, r) .
\end{gathered}
$$

We follow Huang-Krantz's strategy from [7] and we define the following functional

$$
\Omega(\mathcal{F}, X, r)=\left.\frac{q(z(1+\mathcal{F}), X)+P(z(1+\mathcal{F}), X)}{r^{2}}\right|_{\gamma_{X, r}},
$$

where $\mathcal{F}=\mathcal{F}(z, X, r)$. By linearizing in $\mathcal{F}=0$ the functional defined in $(2.27)$, the equation $(2.26)$ becomes

$$
1+\Omega^{\prime}(\mathcal{F}, X, r)+\Omega_{1}(\mathcal{F}, X, r)+\left.\frac{1}{r^{2}} \mathcal{H}_{X, r}[K(z(1+\mathcal{F}), X)]\right|_{\gamma_{X, r}}-\frac{V(X, r)}{r^{2}}=0
$$

where $\mathcal{F}=\mathcal{F}(z, X, r)$ and $\Omega_{1}(\mathcal{F}(z, X, r), X, r)$, are terms that are coming from the Taylor expansion of $P(z, X)$ and

$$
\Omega^{\prime}(\mathcal{F}, X, r)=\left.\frac{2}{r^{2}} \operatorname{Re}\left\{(q+P)_{z}(z, X) z \mathcal{F}\right\}\right|_{\gamma_{X, r}}
$$

We put the normalization condition $V(X, r)=r^{2}$. In order to find a solution $\mathcal{F}$ in the Holder space $\left(\mathcal{C}^{j, \alpha},\|\cdot\|_{j, \alpha}\right)$ for $(2.28)$, we need to study the regularity properties of the functional $\Omega$. We consider the following notation

$$
\mathcal{C}_{X, r}(z)=\left.\frac{2}{r^{2}} \operatorname{Re}\left\{(q+P)_{z}(z, X) z\right\}\right|_{\gamma_{X, r}}
$$

Since $\mathcal{C}_{X, r}(z) \neq 0$ for $|r|<<1, X \approx 0 \in \mathbb{C}^{N-2}$, we can write $\mathcal{C}_{X, r}(z)=$ $\mathcal{A}(z, X, r) \mathcal{B}(z, X, r)$ with

$$
\mathcal{A}(z, X, r)=\left|\mathcal{C}_{X, r}(z)\right|, \quad \mathcal{B}(z, X, r)=\frac{\mathcal{C}_{X, r}(z)}{\left|\mathcal{C}_{X, r}(z)\right|}
$$


Then $\ln \mathcal{B}(z, X, r)$ is a well-defined smooth function in $(z, X, r)$. Among the lines developed by Huang-Krantz in [7], we define the following function

$$
\mathcal{C}^{\star}(z, X, r)=\frac{e^{i \mathcal{H}_{X, r}(\ln \mathcal{B}(z, X, r))}}{\mathcal{A}(z, X, r)} .
$$

Then $\mathcal{C}^{\star}$ is a smooth positive function and $D(z, X, r):=\mathcal{C}^{\star}(z, X, r) \mathcal{C}(z, X, r)$ is holomorphic in $z$, smooth in $(X, r)$. We write $D(z, X, r) \mathcal{F}(z, X, r) \equiv U(z, X, r)+$ $\sqrt{-1} \mathcal{H}_{X, r}[U(z, X, r)]$. Since $D(z, X, r) \neq 0$ we can rewrite $(2.28)$ as follows

$$
\begin{gathered}
U(z, X, r)=-C^{\star}(z, X, r)\left(\Omega_{1}\left(\frac{U(z, X, r)+i \mathcal{H}_{X, r}[U(z, X, r)]}{D(z, X, r)}, X, r\right)\right) \\
-C^{\star}(z, X, r) \frac{1}{r^{2}} \mathcal{H}_{X, r}\left[K\left(z\left(1+\frac{U(z, X, r)+i \mathcal{H}_{X, r}[U(z, X, r)]}{D(z, X, r)}\right), X\right)\right] .
\end{gathered}
$$

We summarize all the precedent computations and we obtain the following regularity result

THEOREM 2.6. The equation (2.33) has a unique solution in the Banach space $\left(\mathcal{C}^{j, \alpha},\|\cdot\|_{j, \alpha}\right)$ such that

$$
\begin{aligned}
\|U\|_{j, \alpha} & =O\left(r^{l-2}\right), \text { for all } j \leq l-2 ; \quad\left\|\left(\partial_{X}^{|I|} \partial_{r}^{s}\right) U\right\|_{j, \alpha} \\
& =O\left(r^{l-s-2}\right), \text { for all } j+2 s \leq l-4, I \in \mathbb{N}^{N-2} .
\end{aligned}
$$

Proof. The solution $U$ and its uniqueness follows by applying the implicit function theorem. We denote by $\Lambda_{1}(U, X, r)$ and $\Lambda_{2}(U, X, r)$ the first and the second term from (2.33). It follows that

$$
\begin{aligned}
\|U\|_{j, \alpha} & \leq\left\|\Lambda_{1}(U, X, r)\right\|_{j, \alpha}+\left\|\Lambda_{2}(U, X, r)\right\|_{j, \alpha} \\
& \leq\left\|\Lambda_{1}(U, X, r)\right\|_{j, \alpha}+\mathrm{O}\left(r^{l-2}\right) \leq C\|U\|_{j, \alpha}^{2}+\mathrm{O}\left(r^{l-2}\right),
\end{aligned}
$$

for some $C>0$. It follows that $\|U\|_{j, \alpha}=\mathrm{O}\left(r^{l-2}\right)$.

The proof of the second regularity property goes after the previous line. Differentiating with $r$ the equation (2.33) it follows that $\partial_{r} U=\partial_{r} \Lambda_{1}(U, X, r)+$ $\partial_{U} \Lambda_{1}(U, X, r)\left[\partial_{r} U\right]+\partial_{r} \Lambda_{2}(U, X, r)+\partial_{U} \Lambda_{2}(U, X, r)\left[\partial_{r} U\right]$. By Proposition 2.3 and Proposition 2.5 we obtain that $\left\|\partial_{r} U\right\|_{j, \alpha}=\mathrm{O}\left(r^{l-2-1}\right)$. Since $P(z, X)=\mathrm{O}\left(z^{3}\right)$ and $K(z, X)=\mathrm{O}\left(z^{l}\right)$, by taking higher derivatives of $x$ in (2.33) it follows that the differentiation of any order with $x \in X$ does not affect the estimates. Therefore the second estimates follow immediately.

We write that

$$
\mathcal{F}_{X, r}\left[\varphi_{X, r}\right]=\frac{U(z, X, r)+i \mathcal{H}_{X, r}[U(z, X, r)]}{D(z, X, r)}:=\varphi_{X, r}+i \mathcal{H}_{X, r}\left[\varphi_{X, r}\right],
$$

where $\left\|\varphi_{X, r}\right\|_{j, \alpha}=\mathrm{O}\left(r^{l-2}\right)$, for all $j \leq l-2$ and $\left\|\left(\partial_{X}^{|I|} \partial_{r}^{s}\right) \varphi_{X, r}\right\|_{j, \alpha}=$ $\mathrm{O}\left(r^{l-s-2}\right)$, for all $j+2 s \leq l-4, I \in \mathbb{N}^{N-2}$. 


\section{A family of analytic discs and proofs of main results.}

3.1. A family of analytic discs. We construct a continuous mapping $T$ defined on $\widetilde{M}_{0}$ into $\mathbb{C}^{2}$ that is holomorphic in $z$ for each fixed $u=r^{2}$ and that maps slice by slice the hypersurface $M_{0}$ into $M$. Let $\varphi_{X, r}$ be the function defined by (2.35). Then

$$
\begin{aligned}
& \mathcal{F}_{X, r}\left[\varphi_{X, r}\right]=\varphi_{X, r}+i \mathcal{H}_{X, r}\left[\varphi_{X, r}\right] \\
& \mathcal{B}_{X, r}\left[\varphi_{X, r}\right]=(q+P+i K)\left(z+z \mathcal{F}_{X, r}\left[\varphi_{X, r}\right], X\right) .
\end{aligned}
$$

We extend these functions to $\widetilde{M}_{0}$ by the Cauchy integral as follows

$$
\begin{aligned}
\mathcal{F}(\zeta, X, r) & =\mathcal{C}\left(\mathcal{F}_{X, r}\left[\varphi_{X, r}\right]\right)(\zeta) \equiv \frac{1}{2 \pi i} \int_{0}^{2 \pi} \frac{\mathcal{F}_{X, r}\left[\varphi_{X, r}\right](\theta) z_{\theta}(\theta, X, r)}{z(\theta, X, r)-\zeta} d \theta \\
\mathcal{B}(\zeta, X, r) & =\mathcal{C}\left(\mathcal{B}_{X, r}\left[\varphi_{X, r}\right]\right)(\zeta) \equiv \frac{1}{2 \pi i} \int_{0}^{2 \pi} \frac{\mathcal{B}_{X, r}\left[\varphi_{X, r}\right](\theta) z_{\theta}(\theta, X, r)}{z(\theta, X, r)-\zeta} d \theta
\end{aligned}
$$

where $z=z(\theta, X, r)$ is a parameterization of the curve $\gamma_{X, r}$ defined by (2.18).

We define $T$ by (3.1). Then $T$ is continuous by construction up to the boundary on each slice $(X, r)=$ constant. In order to obtain the regularity of $T$, we have to bound the derivatives in $(z, X, u)$ of $\mathcal{F}$ and $\mathcal{B}$. We state the following lemma:

Lemma 3.1. For all $j+2 s \leq l-4, I \in \mathbb{N}^{N-2}$ as $r \mapsto 0$, we have

$$
\partial_{\theta}^{j} \partial_{X}^{|I|} \partial_{r}^{s} \mathcal{F}(z, X, r)=O\left(r^{l-s-2}\right), \quad \partial_{z}^{j} \partial_{X}^{|I|} \partial_{r}^{s} \mathcal{B}(z, X, r)=O\left(r^{l-s}\right) .
$$

The proof of the predent Lemma follows by the lines of Lemma 4.1 proof from Kenig-Webster's paper [14].

Theorem 3.2. Let $M$ defined by (2.11) with $P(z, X)=O\left(z^{3}\right), K(z, X)=$ $O\left(z^{l}\right), l \geq 7, T$ extended by (3.2). Then $\widetilde{M}=T\left(\widetilde{M}_{0}\right)$ is a complex manifold-withboundary regularly foliated by discs embedded of class $\mathcal{C}^{\frac{l-7}{3}}$.

Proof. Since $\partial_{u}=\frac{1}{2 r} \partial_{r}$, it follows that

$$
\partial_{z}^{j} \partial_{X}^{|I|} \partial_{u}^{s} \mathcal{F}_{X, r}(z, X, r)=\mathrm{O}\left(r^{l-2 s-j-2}\right), \quad \partial_{z}^{j} \partial_{X}^{|I|} \partial_{u}^{s} \mathcal{F}_{X, r}(z, X, r)=\mathrm{O}\left(r^{l-2 s}\right),
$$

and these derivatives remain bounded for all $j+2 s \leq l-4, I \in \mathbb{N}^{N-2}$. It follows that the jacobian matrix $D T$ of $T=T(X)$ is the identity matrix.

3.2. Proof of Theorem 1.1. Let $M, \widetilde{M}, T$ as in Theorem 3.2. Using the techniques from [16], [19] together with an extended reflection principle as in the paper [15] of Kenig-Webster, we construct smooth extension of $T$ past every point of $M_{0}-\{0\}$. By similar arguments as in the papers [14], [15] of Kenig-Webster, we obtain that $M \cup \widetilde{M}$ is a smooth manifold-with-boundary $M$ in a neighborhood of the CR singular point $p=0$.

3.3. Proof of Theorem 1.3. Since the hypersurface given by Theorem 2.1 is Levi-flat it follows each of our analytic discs is a reparameterization of an analytic disc contained inside. By dimension reasons it follows that the under the hypothesis of Theorem 1.2, the hypersurfaces given by Theorem 1.1 and Theorem 1.2 are the same. 
3.4. Proof of Theorem 1.4. We can study now the hull of $M$ near $p=0$ when $M$ is assumed to be real-analytic. The hypersurface $M_{0}$ defined by $(2.23)$ is foliated by a the family of analytic discs defined by $(2.19)$ and therefore $\widetilde{M}$ is foliated by the family of analytic discs defined by (3.2). By similar arguments as in Section 7 of the paper [13] of Huang-Yin we obtain our result. The author believes that the arguments from the paper [7] of Huang-Krantz or from the paper [8] of Huang can be adapted in order to prove the analyticity in our case.

\section{REFERENCES}

[1] E. Bishop, Differentiable Manifolds In Complex Euclidian Space, Duke Math. J., 32:1 (1965), pp. 1-21.

[2] P. Dolbeault, G. Tomassini, and D. Zaitsev, On Levi-flat hypersurfaces with prescribed boundary, Pure and Applied Mathematics Quarterly, 6:3 (2010), (Special Issue: In honor of Joseph J. Kohn. Part 1), pp. 725-753.

[3] P. Dolbeault, G. Tomassini, and D. Zaitsev, Boundary Problem for Levi-Flat Graphs, Indiana Univ. Math. J., 60:1 (2011), pp. 161-170.

[4] X. Gong, Existence of real analytic surfaces with hyperbolic complex tangent that are formally but not holomorphically equivalent to quadrics, Indiana Univ. Math. J., 53:1 (2004), pp. 8395.

[5] L. Helmes, Potential Theory, Spriger-Verlag London, (2009).

[6] L. Hormander, An introduction to Complex Analysis in Several Variables, Second Revisited edition, North-Holland Library, Vol. 7., North-Holland Publishing Co., AmsterdamLondon; American Elvesier Publishing Co., Inc., New York, (1973).

[7] X. Huang and S. Krantz, On a problem of Moser, Duke Math. J., 78:1 (1995), pp. 213-228.

[8] X. Huang, On a n-manifold in $\mathbb{C}^{n}$ near an elliptic complex tangent, J. Amer. Math. Soc., 11:3 (1998), pp. 669-692.

[9] X. Huang, Local Equivalence Problems for Real Submanifolds in Complex Spaces, Lecture Notes in Mathematics, Spriger-Verlag, pp. 109-161, Berlin-Heidelberg-New York, (2004).

[10] X. Huang And W. Yin, A codimension two CR singular submanifold that is formally equivalent to a symetric quadric, Int. Math. Res. Not. IMRN, 15 (2009), pp. 2789-2828.

[11] X. Huang And W. Yin, A Bishop surface with a vanshing Bishop invariant, Invent. Math., 176:3 (2010), pp. 461-520.

[12] X. Huang And W. Yin, Equivalence problem for Bishop surfaces, Sci. China Math., 43:3 (2010), pp. $687-700$.

[13] X. HUANG AND W. Yin, Flattening of CR singular points and the analicity of the local hull of holomorphy, preprint 2012.

[14] C. Kenig and S. Webster, On the local hull of holomorphy of an n-manifold in $\mathbb{C}^{n}$, Ann. Scuola Norm. Sup. Pisa Cl. Sci., 19:2 (1984), pp. 261-280.

[15] C. Kenig And S. Webster, The hull of holomorphy of a surface in the space of two complex variables, Inv. Math., 19:2 (1982), pp. 261-280.

[16] J. MATHER, On Nirenberg's proof of Malgrange's preparation theprem, Proceedings of Liverpool Singularities Symposium 1, Lec. Notes in Math., Springer-Verlag, 192 (1971), pp. 116-120.

[17] J. Moser, Analytic Surfaces in $\mathbb{C}^{2}$ and Their Local Hull of Holomorphy, Ann. Acad. Sci. Fenn. Ser. A Math., 10 (1985), pp. 397-410.

[18] J. Moser and S. Webster, Normal forms for real surfaces in $\mathbb{C}^{2}$ near complex tangents and hyperbolic surface tranformations, Acta Math., 150 (1983), pp. 255-296.

[19] L. Nirenberg, S. Webster, And P. Yang, Local Boundary Regularity of Holomorphic Mappings, Comm. Pure Appl. Math., 33 (1980), pp. 305-338.

[20] J. LeBl, Nowhere minimal CR submanifolds and Levi-flat hypesurfaces, J. Geom. Anal., 17:2 (2007), pp. 321-341. 